\title{
Cystathionine $\beta$-synthase (CBS) deficiency suppresses erythropoiesis by disrupting expression of heme biosynthetic enzymes and transporter
}

\author{
Peng Zhao ${ }^{1,2}$, Christopher Qian ${ }^{3}$, Yun-Jin Chen ${ }^{1,2}$, Yuan Sheng ${ }^{1,2}$, Ya Ke $e^{3}$ and Zhong-Ming Qian (1) ${ }^{1,2,4}$
}

\begin{abstract}
The reduced iron usage induced by the suppression of erythropoiesis is a major cause of the systemic iron overload in CBS knockout $\left(\mathrm{CBS}^{-1-}\right)$ mice. However, the relevant mechanisms are unknown. Here, we examined changes in granulocyte/erythroid cell ratios, iron content, and expression of iron-metabolism proteins, including; two key enzymes involved in the heme biosynthetic pathway, ALAS2 (delta-aminolevulinate synthase 2) and FECH (ferrochelatase), a heme exporter from the cytosol and mitochondria, FLVCR (feline leukemia virus subgroup C cellular receptor) as well as EPO (erythropoietin), EPOR (erythropoietin receptor) and HIF-2a (hypoxia inducible factor-2 subunit a), in the blood, bone marrow or liver of $\mathrm{CBS}^{-/-}$(homozygous), $\mathrm{CBS}^{+/-}$(heterozygous) and $\mathrm{CBS}^{+/+}$(Wild Type) mice. Our findings demonstrate that CBS deficiency can induce a significant reduction in the expression of ALAS2, FECH, FLVCR, HIF-2a, EPO, and EPOR as well as an increase in interleukin-6 (IL-6), hepcidin and iron content in the blood, bone marrow or liver of mice. We conclude that the suppression of erythropoiesis is mainly due to the CBS deficiencyinduced disruption in the expression of heme biosynthetic enzymes and heme-transporter.
\end{abstract}

In addition to CSE (cystathionine gammalyase) ${ }^{1}$ and MST (3-mercaptopyruvate sulfurtransferase) $)^{2,3}$, CBS (Cystathionine $\beta$ - synthase) is a key enzyme for hydrogen sulfide (H2S) production from L-cysteine ${ }^{4,5}$. $\mathrm{CBS}^{-1-}$ (homozygous knockout) mice rarely survive past 4 weeks of age ${ }^{4}$, implying that CBS is essential for survival and development in mice. In a recent study, we found that CBS knockout $\left(\mathrm{CBS}^{-1-}\right)$ mice exhibited anemia and a significant increase in iron content in the serum, liver, spleen, and heart, along with severe damage to the liver, displaying a hemochromatosis-like phenotype ${ }^{6}$. Also, we demonstrated that this iron-related phenotype could

\footnotetext{
Correspondence: Ya Ke (keyacuhk@sina.com) or Zhong-Ming Qian (qianzhongming@fudan.edu.cn)

'Laboratory of Neuropharmacology, Fudan University School of Pharmacy, Shanghai 201203, China

${ }^{2}$ National Clinical Research Center for Aging and Medicine, Huashan Hospital, FudanUniversity, Shanghai 200040, China

Full list of author information is available at the end of the article.

These authors contributed equally: Ya Ke and Zhong-Ming Qian

Edited by A. Finazzi-Agrò
}

partially be reversed by administration of CBSoverexpressing adenovirus. These findings showed that CBS is required for body iron homeostasis ${ }^{6}$.

The reduced iron usage due to suppressed erythropoiesis is a major cause of the systemic iron overload ${ }^{6}$. However, the molecular mechanisms by which CBS deficiency suppresses erythropoiesis are unknown. In this study, we first examined in detail the effects of $\mathrm{CBS}^{-/-}$on red blood cell (RBC), hematocrit (HCT), hemoglobin $(\mathrm{Hb})$, mean corpuscular hemoglobin $(\mathrm{MCH})$, mean corpuscular volume (MCV), iron, interleukin-6 (IL-6), and hepcidin contents in the blood, and the ratio of granulocyte/erythroid cells, iron, ferritin-light chain (Ft-L) and ferritin-heavy chain (Ft-H) levels, as well as deltaaminolevulinate synthase 2 (ALAS2) and ferrochelatase $(\mathrm{FECH})$, two key enzymes involved in the heme biosynthetic pathway, and feline leukemia virus subgroup $\mathrm{C}$ cellular receptor (FLVCR), a heme exporter from the cytosol and mitochondria in the bone marrow of $\mathrm{CBS}^{-1-}$

\section{(c) The Author(s) 2019}

(c) (i) Open Access This article is licensed under a Creative Commons Attribution 4.0 International License, which permits use, sharing, adaptation, distribution and reproduction cc) in any medium or format, as long as you give appropriate credit to the original author(s) and the source, provide a link to the Creative Commons license, and indicate if changes were made. The images or other third party material in this article are included in the article's Creative Commons license, unless indicated otherwise in a credit line to the material. If material is not included in the article's Creative Commons license and your intended use is not permitted by statutory regulation or exceeds the permitted use, you will need to obtain permission directly from the copyright holder. To view a copy of this license, visit http://creativecommons.org/licenses/by/4.0/. 
(homozygous), $\mathrm{CBS}^{+/-}$(heterozygous), and $\mathrm{CBS}^{+/+}$(Wild Type) mice.

We then investigated the expression of iron transport proteins, including transferrin receptor 1 (TfR1), divalent metal transporter 1 (DMT1), ferroportin 1 (Fpn1), iron storage proteins $\mathrm{Ft}-\mathrm{L}$ and $\mathrm{Ft}-\mathrm{H}$, iron regulatory hormone (hepcidin) and proteins (IRPs), and other relevant molecules, including erythropoietin (EPO) and its receptor (EPOR), hypoxia inducible factor-2 subunit $\alpha$ (HIF-2 $\alpha$ ), phosphorylated Janus kinase 2 (p-JAK2) and erythroferrone (ERFE) in the bone marrow, bone marrowderived macrophages (BMDMs) or liver of $\mathrm{CBS}^{-1-}, \mathrm{CBS}^{+/-}$, and $\mathrm{CBS}^{+/+}$mice.

Our findings demonstrated that CBS deficiency could induce a significant reduction in the expression of ALAS2, $\mathrm{FECH}$, and FLVCR, alongside a significant increase in iron content in the bone marrow, indicating that the suppression of erythropoiesis is mainly due to the inhibitory effects of CBS deficiency on two key enzymes involved in the heme biosynthetic pathway, and also on the transport of heme from the cytosol and mitochondria. The reduced expression of EPO in $\mathrm{CBS}^{-1-}$ mice could lead to an increase in CFU-E (colony-forming unit erythroids) apoptosis and hence reduce the number of erythroblasts, which may also be one of the molecular mechanisms involved in the suppression of erythropoiesis in $\mathrm{CBS}^{-1-}$ mice.

\section{Results}

CBS deficiency induced a reduction in $\mathrm{RBC}, \mathrm{Hb}$, and $\mathrm{HCT}$, and an increase in $\mathrm{MCH}, \mathrm{MCV}$, iron, IL-6, and hepcidin in the blood

To find out the effects of CBS deficiency on erythropoiesis, we initially measured $\mathrm{RBC}, \mathrm{Hb}, \mathrm{HCT}, \mathrm{MCH}$, MCV and also the contents of iron, IL- 6 and hepcidin in the blood of $\mathrm{CBS}^{+/+}, \mathrm{CBS}^{+/-}$, and $\mathrm{CBS}^{-1-}$ mice. The contents of RBC (Fig. 1a), Hb (Fig. 1b), and HCT (Fig. 1c) were found to be significantly lower, while the levels of $\mathrm{MCH}$ (Fig. 1d) and MCV (Fig. 1e) higher in $\mathrm{CBS}^{-1-}$ mice when compared to $\mathrm{CBS}^{+/+}$and $\mathrm{CBS}^{+/-}$mice. There were no statistically significant differences in these measurements between $\mathrm{CBS}^{+/+}$and $\mathrm{CBS}^{+/-}$mice. The findings
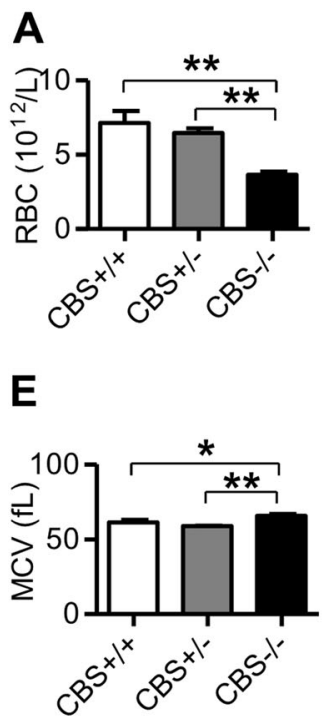

$\mathbf{F}$

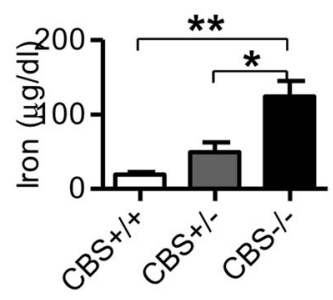

I

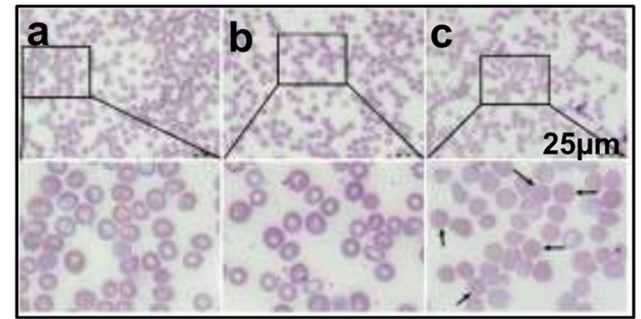

C

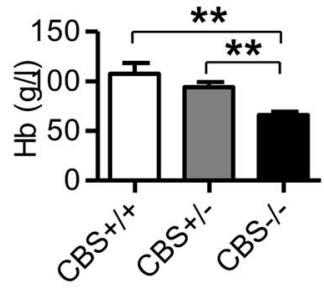

G

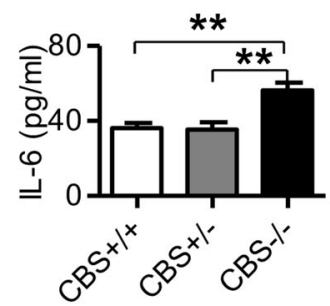

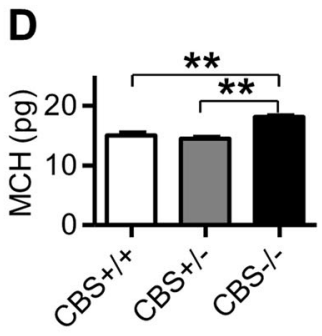

H

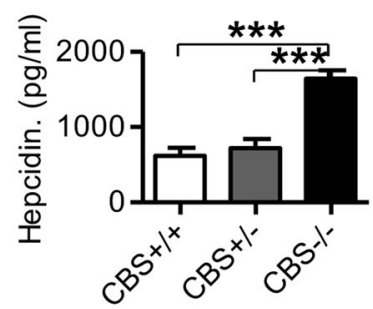

Fig. 1 CBS deficiency induced a reduction in $\mathrm{RBC}, \mathrm{Hb}$, and $\mathrm{HCT}$, and an increase in $\mathrm{MCH}, \mathrm{MCV}$, iron, IL-6, and hepcidin in the blood. RBC (a), $\mathrm{Hb}(\mathbf{b}), \mathrm{HCT}(\mathbf{c}), \mathrm{MCH}(\mathbf{d}), \mathrm{MCV}(\mathbf{e})$, iron $(\mathbf{f}), \mathrm{IL}-6(\mathbf{g})$ and hepcidin $(\mathbf{h})$ in the blood and blood smear $(\mathrm{l}, \times 1000)$ of CBS ${ }^{+/+}(\mathbf{a}), \mathrm{CBS}^{+/-}(\mathbf{b})$ and $\mathrm{CBS}{ }^{-/-}(\mathbf{c})$ mice were measured as described in the "Materials and Methods" section. Data are means \pm SEM $(n=6) .{ }^{*} p<0.05,{ }^{* *} p<0.01,{ }^{* * *} p<0.001 \mathrm{vs}$. CBS ${ }^{+/+}$ or $\mathrm{CBS}^{+/-}$mice 
were consistent with the changes in erythrocyte morphologic characteristics (Fig. 1i). Observation of blood smear showed that the erythrocytes were uniform in size in $\mathrm{CBS}^{+/+}$mice (Fig. 1Ia), whereas the central pale area of erythrocytes was enlarged and annular erythrocytes were visible in $\mathrm{CBS}^{+/-}$mice (Fig. 1Ib), and in $\mathrm{CBS}^{-1-}$ mice, the central pale area of erythrocytes was reduced or largely disappeared and spherical erythrocytes were visible (Fig. 1Ic). These findings demonstrated that CBS deficiency could induce a significant reduction in $\mathrm{RBC}, \mathrm{Hb}, \mathrm{HCT}$, an increase in $\mathrm{MCH}$ and $\mathrm{MCV}$, and the formation of spherical erythrocytes. In addition, the contents of iron (Fig. 1f), IL-6 (Fig. 1g) and hepcidin (Fig. 1h) in the blood of
$\mathrm{CBS}^{-1-}$ mice were significantly higher than those in the $\mathrm{CBS}^{+/+}$and $\mathrm{CBS}^{+/-}$mice.

CBS deficiency increased the ratio of granulocyte/erythroid cells, iron, $\mathrm{Ft}-\mathrm{L}$ and $\mathrm{Ft}-\mathrm{H}$ contents, and reduced the expression of ALAS2, FECH, and FLVCR mRNAs in the bone marrow

We then examined the changes occurring in the bone marrow induced by CBS deficiency, and found that there was no abnormal cell morphology in bone marrow smear of $\mathrm{CBS}^{+/+}$mice (Fig. 2Aa). There was no significant difference in the observed bone marrow smear between the $\mathrm{CBS}^{+/+}$mice (Fig. 2Aa) and $\mathrm{CBS}^{+/-}$mice
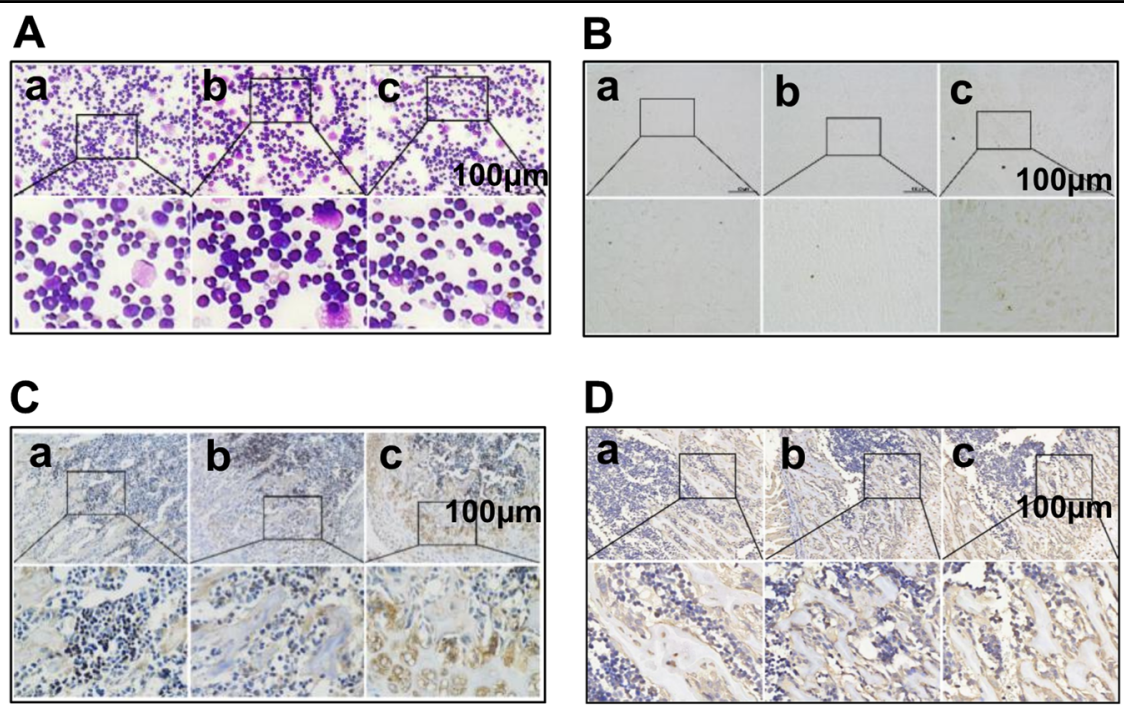

E
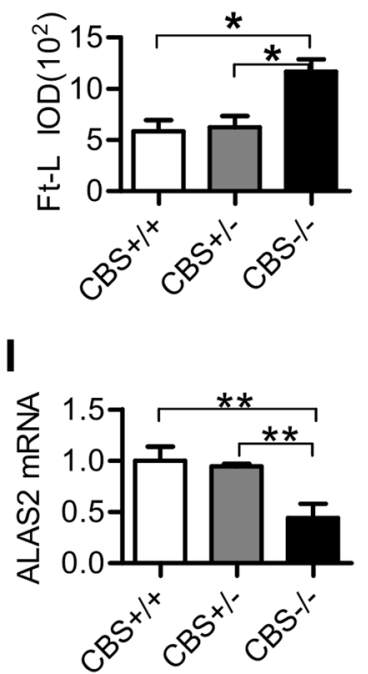

$\mathbf{F}$

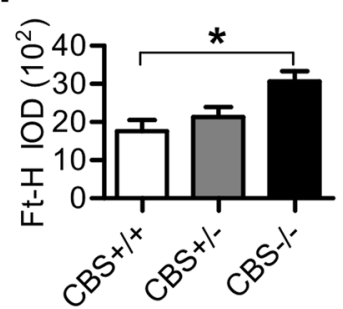

J

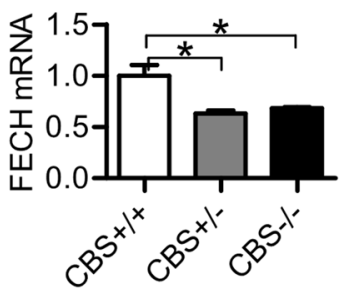

G

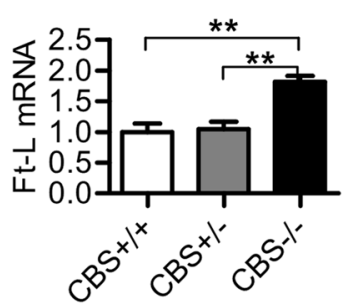

K

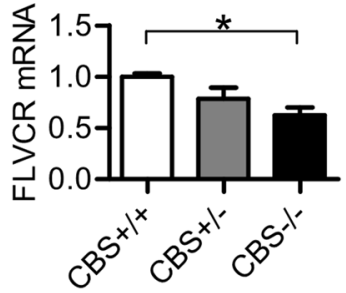

H

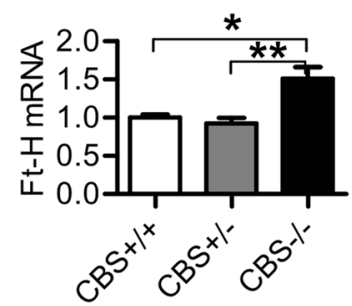

Fig. 2 CBS deficiency increased the ratio of granulocyte/erythroid cells, iron, Ft- $\mathrm{L}$, and Ft-H contents, and reduced expression of ALAS2, FECH, and FLVCR mRNAs in the bone marrow. a Bone marrow smear (granulocyte/erythroid cells); $\mathbf{b}$ Prussian blue staining (iron) in the bone marrow; $\mathbf{c}-\mathbf{j}$ Immunofluorescence analysis of the expression of Ft-L (c and $\mathbf{e}$ ) and Ft-H (d and $\mathbf{f}$ ) proteins in the bone marrow; and G-K: Real-time PCR analysis of the expression of $\mathrm{Ft}-\mathrm{L}(\mathbf{g}), \mathrm{Ft}-\mathrm{H}(\mathbf{h}), \mathrm{ALAS2}(\mathbf{i}), \mathrm{FECH}(\mathbf{j})$ and FLVCR $(\mathrm{K})$ mRNAs in the bone marrow of CBS ${ }^{+/+}(\mathbf{a}), \mathrm{CBS}^{+/-}(\mathbf{b})$, and $\mathrm{CBS}{ }^{-/-}(\mathbf{c})$ mice. Data are means \pm SEM $(n=6) .{ }^{*} p<0.05,{ }^{* *} p<0.01$ vs. $\mathrm{CBS}^{+/+}$or $\mathrm{CBS}^{+/-}$mice 
(Fig. 2Ab). However, the ratio of granulocyte/erythroid cells significantly increased and the number of erythroid cells at each stage was reduced in $\mathrm{CBS}^{-1-}$ mice when compared with $\mathrm{CBS}^{+/+}$mice (Fig. 2Ac, Supplementary Table 2 and 3).

As Iron is a hematopoietic raw material ${ }^{7}$, we therefore investigated the effect of CBS deficiency on iron content in the bone marrow. Prussian blue staining demonstrated that iron levels in the bone marrow were significantly higher in $\mathrm{CBS}^{-1-}$ mice (Fig. 2Bc) than in $\mathrm{CBS}^{+/-}$ (Fig. 2Bb) and $\mathrm{CBS}^{+/+}$mice (Fig. 2Ba). Also, the number of sideroblasts was significantly increased in the bone marrow of $\mathrm{CBS}^{-1-}$ mice as compared with $\mathrm{CBS}^{+/-}$and $\mathrm{CBS}^{+/+}$mice (Supplementary Table 4 and 5), and the percentage of type IV sideroblasts was remarkably higher in $\mathrm{CBS}^{-1-}$ mice (Supplementary Fig. 1c) than in CBS ${ }^{+/-}$ (Supplementary Fig. 1b) and $\mathrm{CBS}^{+/+}$mice (Supplementary Fig. 1a), demonstrating that CBS deficiency could induce a significant increase in the number of sideroblasts and iron content in erythroblasts of bone marrow (Supplementary Table 4 and 5, and Supplementary Fig. 1). Western blot and RT-PCR analysis revealed a significant increase in Ft-L and Ft-H Proteins (Ft-L: Fig. 2c, e; Ft-H: Fig. 2d, f) and mRNAs (Ft-L: Fig. 2g; Ft-H: Fig. 2h) in the bone marrow of $\mathrm{CBS}^{-1-}$ mice, as compared with $\mathrm{CBS}^{+/+}$ and $\mathrm{CBS}^{+/-}$mice.

ALAS2 and FECH are the first (rate-limiting) and the last enzymes in the heme biosynthetic pathway, and FLVCR is a heme exporter from the cytosol and mitochondria. We therefore decided to investigate the effect of CBS deficiency on the expression of these molecules in the bone marrow. We did not find any differences in the expression of ALAS2 mRNA between $\mathrm{CBS}^{+/+}$and $\mathrm{CBS}^{+/-}$ mice; however, expression of ALAS2 mRNA was shown to be significantly lower in $\mathrm{CBS}^{-/-}$mice than in $\mathrm{CBS}^{+/+}$and $\mathrm{CBS}^{+1-}$ mice (Fig. 2i). The expression of FECH mRNA was significantly lower in $\mathrm{CBS}^{-1-}$ and $\mathrm{CBS}^{+/-}$mice than in $\mathrm{CBS}^{+/+}$mice (Fig. 2j). A significant reduction was also found in the expression of FLVCR mRNA in the bone marrow of $\mathrm{CBS}^{-/-}$mice, when compared with $\mathrm{CBS}^{+/-}$ mice.

CBS deficiency reduced the expression of TfR1, DMT1, Fpn1, and p-JAK2 proteins and mRNAs, and EPO, EPOR, and ERFE MRNAs in the bone marrow

We also examined the expression of iron uptake proteins TfR1 and DMT1, and iron release protein Fpn1 in the bone marrow. Immunofluorescence and real-time PCR analysis showed that the expression of TfR1 (Fig. 3a, d, and e), DMT1 (Fig. 3b, f, and g) and Fpn1 (Fig. 2c, h, and i) proteins and mRNAs in the bone marrow was significantly lower in $\mathrm{CBS}^{-/-}$mice than in $\mathrm{CBS}^{+/+}$and/or $\mathrm{CBS}^{+/-}$mice. The expression of proteins involved in celliron uptake and release is affected by p-JAK2 (IL-6/p-
JAK2/p-STAT3 pathway), EPO/EPOR and ERFE, and we therefore examined the effects of CBS deficiency on the expression of these molecules. Immunohistochemistry staining and real-time PCR analysis showed that the levels of p-JAK2 protein (Fig. 3j, k), EPO (Fig. 3l), EPOR (Fig. 3m), and ERFE (Fig. 3n) mRNAs in bone marrow in $\mathrm{CBS}^{-1-}$ mice were significantly lower than those in $\mathrm{CBS}^{+/+}$and $\mathrm{CBS}^{+/-}$mice, demonstrating that CBS deficiency could down-regulate the expression of p-JAK2, EPO, EPOR, and ERFE in bone marrow.

CBS deficiency up-regulated the expression of TfR1, Ft-L and $\mathrm{Ft}-\mathrm{H}$ proteins and down-regulated Fpn1 and DMT1 in BMDMs

Because the macrophage plays key functions in heme synthesis $^{8,9}$, we also assessed the changes in the expression of TfR1, DMT1, Fpn1, Ft-L, and Ft-H in the BMDMs of $\mathrm{CBS}^{+/+}, \mathrm{CBS}^{+/-}$, and $\mathrm{CBS}^{-1-}$ mice. Western blot analysis demonstrated that the expression of TfR1 (Fig. 4a), Ft-L (Fig. 4d), and Ft-H (Fig. 4e) proteins was significantly higher, while DMT1 (Fig. 4b) and Fpn1 (Fig. 4c) proteins lower in BMDM cells in $\mathrm{CBS}^{-1-}$ mice, when compared to $\mathrm{CBS}^{+/+}$and $\mathrm{CBS}^{+/-}$mice.

\section{CBS deficiency down-regulated expression of HIF-2a, EPO, and IRPs in the liver}

To further elucidate the mechanisms involved in the suppression of erythropoiesis induced by CBS deficiency, we also investigated the expression of EPO, the upstream regulator HIF- $\alpha$ and IRPs in the liver. Western blot analysis showed that, in addition to the significantly reduced CBS expression (Fig. 5a), CBS deficiency induced a significant reduction in the expression of HIF-2 $\alpha$ (Fig. 5b) and EPO (Fig. 5c) in the liver, being significantly lower in $\mathrm{CBS}^{-/-}$mice when compared to $\mathrm{CBS}^{+/+}$and $\mathrm{CBS}^{+/-}$mice. There were no significant differences in the expression of HIF- $2 \alpha$ and EPO between $\mathrm{CBS}^{+/+}$and $\mathrm{CBS}^{+/-}$mice. These findings were also confirmed by immunofluorescence observation (Fig. 5d). In addition, real-time PCR analysis demonstrated that the expression of IRP1 (Fig. 5e) and IRP 2 (Fig. 5f) mRNAs in the liver of $\mathrm{CBS}^{-1-}$ mice was significantly lower than that in $\mathrm{CBS}^{+/+}$ and $\mathrm{CBS}^{+/-}$mice, implying that $\mathrm{CBS}$ deficiency could also down-regulate the expression of IRP1 and IRP2 in the liver.

\section{Discussion}

In this study, we demonstrated that the absence of CBS $\left(\mathrm{CBS}^{-1-}\right)$ can induce a significant reduction in red blood cell, hemoglobin, and hematocrit in peripheral blood, as well as increase the ratio of granulocyte/erythroid cells with reduced numbers of erythroid cells at all stages in the bone marrow of mice. These findings provide solid evidence for $\mathrm{CBS}^{-1-}$ having a role in suppressing 

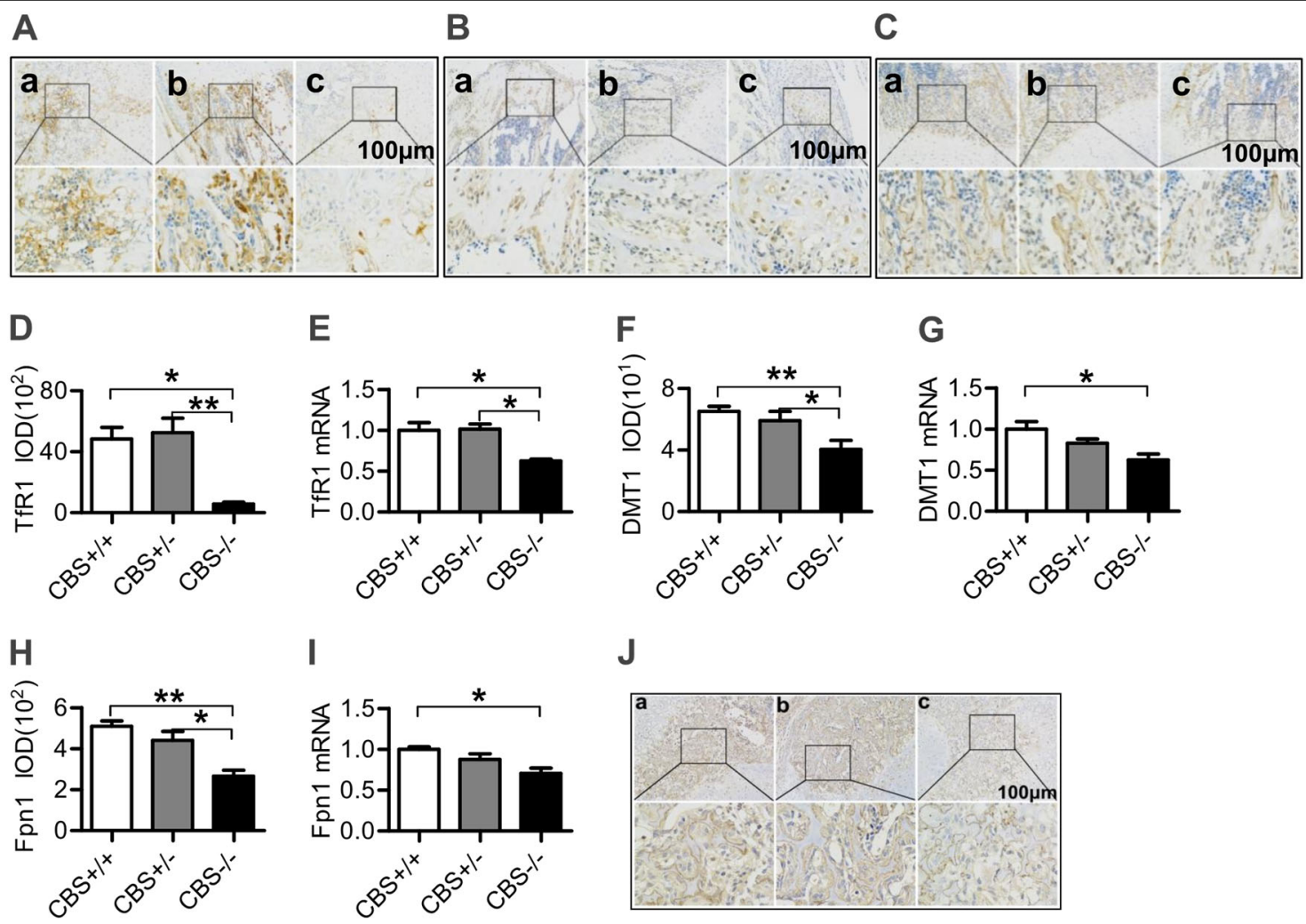

|

$J$
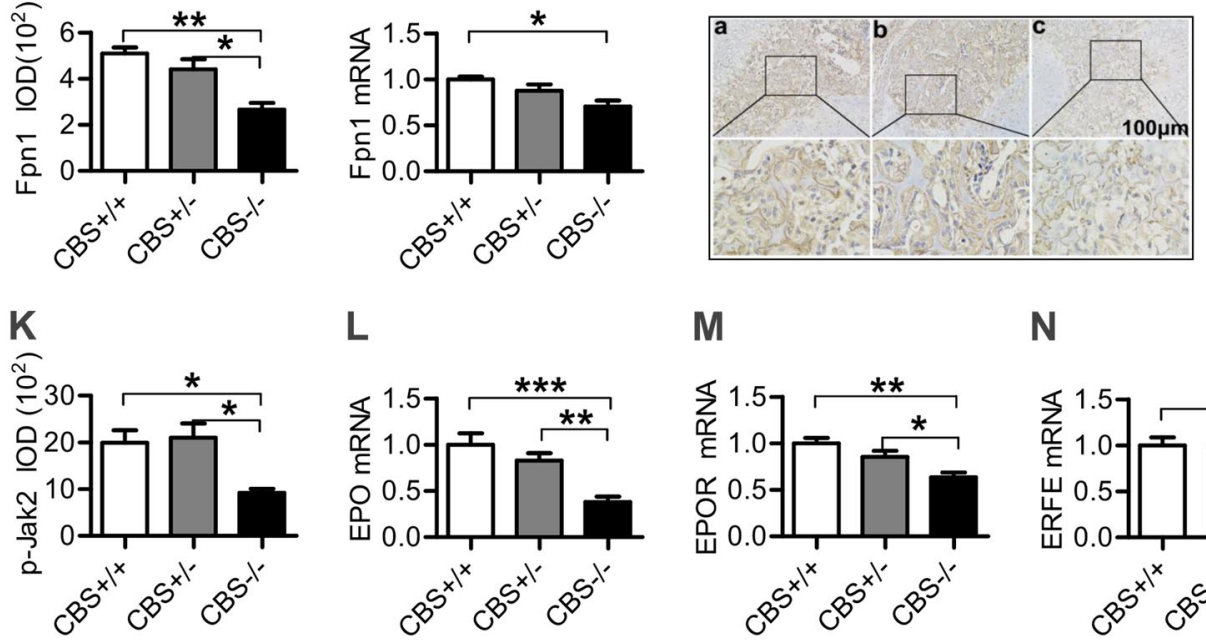

M

$\mathbf{N}$
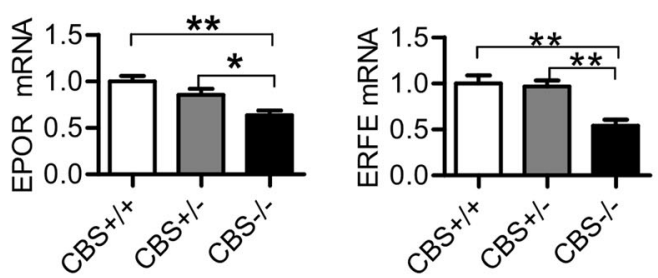

Fig. 3 CBS deficiency reduced expression of TfR1, DMT1, Fpn1 and p-JAK2 Proteins and mRNAs and EPO, EPOR, and ERFE mRNAs in the bone marrow. The expression of TfR1 ( $\mathbf{a}$ and $\mathbf{d})$, DMT1 ( $\mathbf{b}$ and $\mathbf{f}$ ), Fpn1 (c and $\mathbf{h}$ ) and pJAK2 (j and $\mathbf{k})$ proteins and TfR1 (e), DMT1 (g), Fpn1 (i), EPO (I), EPOR $(\mathbf{m})$, ERFE (N) mRNAs in the bone marrow of $\mathrm{CBS}^{+/+}(\mathbf{a}), \mathrm{CBS}^{+/-}(\mathbf{b})$ and $\mathrm{CBS}^{-/-}(\mathbf{c})$ mice was detected by immunohistochemistry staining and

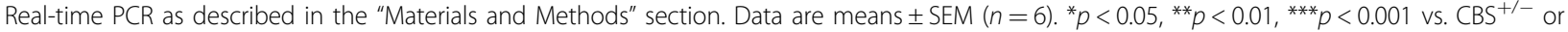
$\mathrm{CBS}^{+/+}$mice

hemoglobin synthesis and erythropoiesis, indicating that CBS is essential for these physiological activities.

Erythropoiesis (erythroid production process) is a tightly regulated and multi-step process, originating in the bone marrow from a multipotent hematopoietic stem cell (HSC) and terminating in a mature, enucleated red blood cell $^{10-12}$. During terminal erythropoiesis, iron is essential for hemoglobin synthesis ${ }^{7}$. Intracellular iron is targeted to the mitochondria for incorporation into a porphyrin ring to form heme and cytosolic iron-sulfur proteins ${ }^{13}$. In mammals, eight enzymes are involved in the heme biosynthetic pathway, located successively in the mitochondria, the cytosol and the mitochondria. ALAS, the first enzyme of the pathway which catalyze the condensation of glycine and succinyl CoA to form delta-aminolevulinic acid (ALA), plays a critical role in controlling the heme biosynthesis ${ }^{14}$. FECH is the last enzyme of the pathway, located in the mitochondrial inner membrane and catalyzes the insertion of $\mathrm{Fe}(\mathrm{II})$ iron into protoporphyrin IX $(\mathrm{PPIX})^{14}$. FLVCR is an exporter of heme from the cytosol and mitochondria ${ }^{15,16}$.

In this study, we found that $\mathrm{CBS}^{-1-}$ could induce a significant reduction in the expression of ALAS1, FECH, and FLVCR and an increase in the iron content of erythroblasts and bone marrow. These findings imply that CBS deficiency suppresses erythropoiesis, mainly by inhibiting expression of heme biosynthetic enzymes and transporters, disrupting heme biosynthesis and reducing 

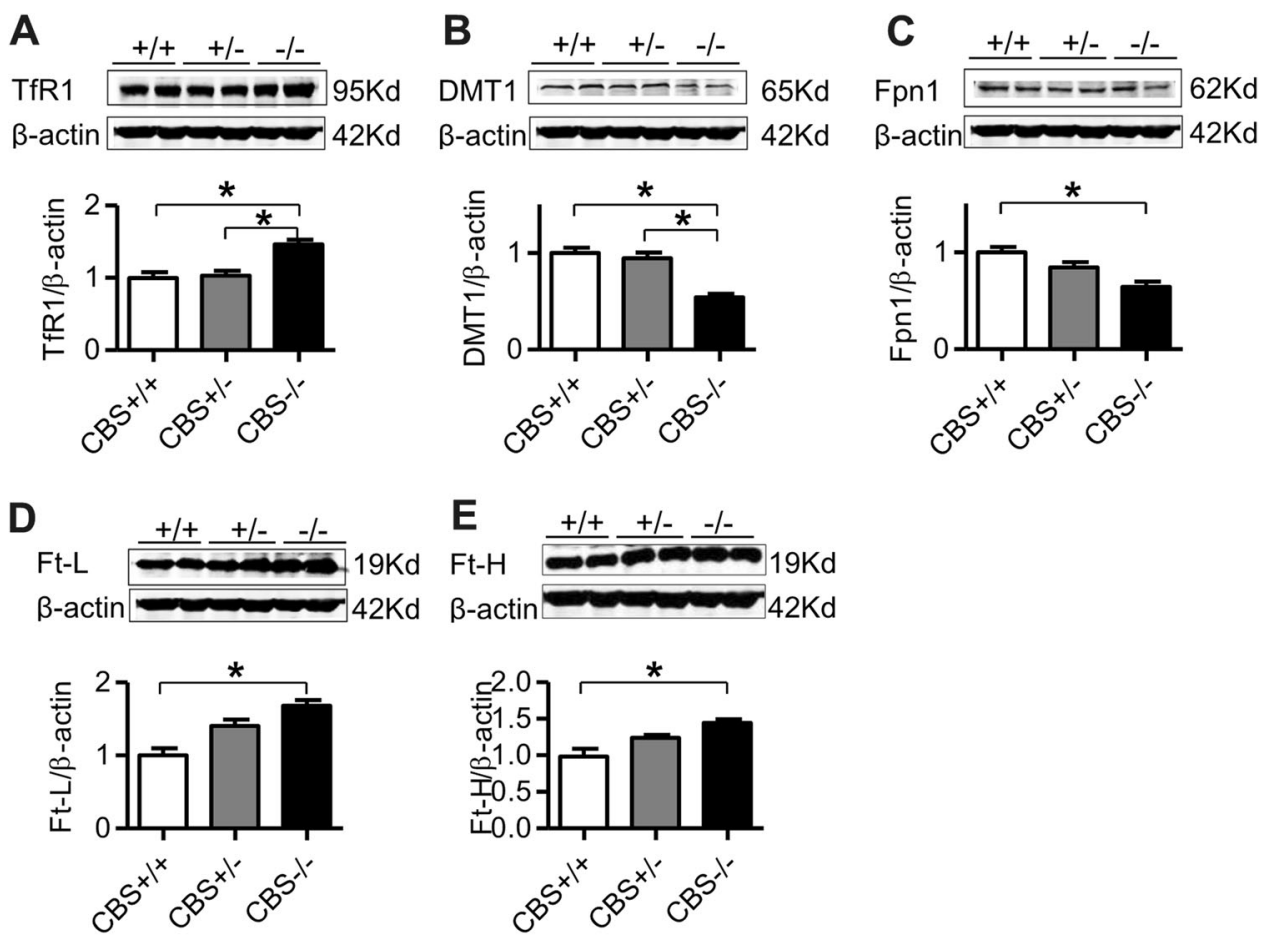

Fig. 4 CBS deficiency up-regulated TfR1 and Fts and down-regulated Fpn1 and DMT1 expression in BMDMs. The expression of TfR1 (a), DMT1 (b), Fpn1 (c), Ft-L (d) and Ft-H (e) in the BMDMs of $\mathrm{CBS}^{+/+}, \mathrm{CBS}^{+/-}$, and $\mathrm{CBS}^{-1-}$ mice was measured by Western blot analysis as described in the "Materials and Methods" section. Data are means \pm SEM $(n=6) .{ }^{*} p<0.05,{ }^{* *} p<0.01,{ }^{* * *} p<0.001$ vs. CBS ${ }^{+/-}$or CBS ${ }^{+/+}$mice

heme export from the cytosol and mitochondria (Fig. 6). The significant increase in iron content of the erythroblasts and bone marrow may mainly be due to the reduction of iron utilization induced by disrupted heme biosynthesis and export, and may also be one of the reasons for the reduced expression of IRP1 and TfR1 in the bone marrow (Fig. 6).

Terminal erythropoiesis occurs in anatomic niches known as erythroblastic islands ${ }^{12}$. Erythroblastic islands are unique to mammalian erythropoiesis and consist of a central macrophage surrounded by up to 30 erythroid cells at varying degrees of red cell maturation ${ }^{17}$. The cells range from colony-forming unit-erythroid cells (CFU-Es) to enucleating erythroblasts, and are the site of hemoglobin synthesis by terminally differentiating erythroblasts $^{12,18}$. It has been well documented that CFU-Es express abundant EPOR ${ }^{19}$. CFU-Es and their progeny divide several times generating 8-64 erythroblasts within 7-8 days in the presence of EPO, whereas CFU-Es undergo apoptosis in the absence of $\mathrm{EPO}^{20}$. HIF-2 $\alpha$ (also known as EPAS1, endothelial PAS domain protein 1 or HLF, HIF-like factor) is the main regulator of EPO production ${ }^{21-}$ ${ }^{23}$. The expression of HIF- $2 \alpha$ has been identified in endothelial cells, hepatocytes, and renal peritubular interstitial cells $^{24,25}$. Renal and hepatic EPO synthesis is predominantly HIF-2 $\alpha$-regulated in response to hypoxia ${ }^{21,25,26}$. The significant reduction in EPO and EPOR/JAK2 found in the bone marrow and liver, and in HIF-2 $\alpha$ in the liver of $\mathrm{CBS}^{-/-}$ mice suggests that CBS deficiency can inhibit EPO expression by a HIF-2 $\alpha$-mediated process and that the reduced expression in EPO may increase apoptosis of CFU-Es and reduce the number of erythroblasts, which may also contribute to the suppressed erythropoiesis in CBS deficient mice (Fig. 6).

Inflammation has been demonstrated to negatively affect the synthesis and biological activity of $\mathrm{EPO}^{27}$, due to the combination of direct inhibition via cytokines ${ }^{28}$ as well as the expression of EPOR on erythroid progenitors ${ }^{29,30}$. It has also been demonstrated that $\mathrm{CBS}^{-1-}$ can up-regulate the expression of IL-6 in the liver ${ }^{6}$. In this study, we demonstrated that EPO and EPOR expression in bone marrow (erythroid progenitors) is significantly reduced, while IL-6 in serum is increased in $\mathrm{CBS}^{-1-}$ mice. These findings indicate that in addition to HIF- $2 \alpha$, the increased inflammatory cytokine IL- 6 may also be associated with the reduced expression of EPO and EPOR, and subsequently ineffective erythropoiesis in $\mathrm{CBS}^{-1-}$ mice (Fig. 6).

In addition to the reduced iron utilization, the increased iron content in erythroblasts and bone marrow may be partly associated with the reduced Fpn1 expression and iron release from bone marrow cells in $\mathrm{CBS}^{-1-}$ mice. Fpn1 is the only identified cellular iron exporter, and is also a receptor 

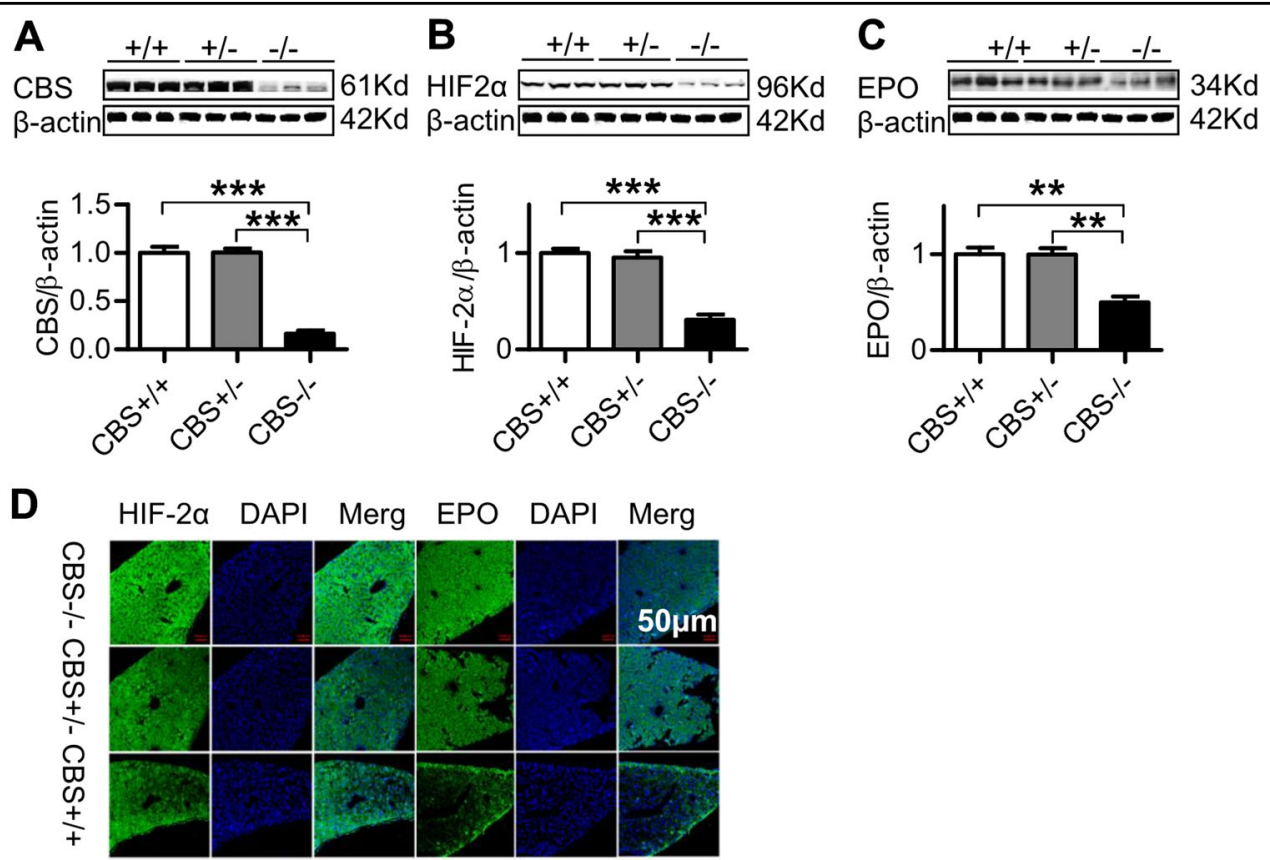

E

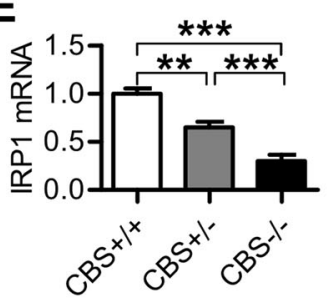

$\mathbf{F}$

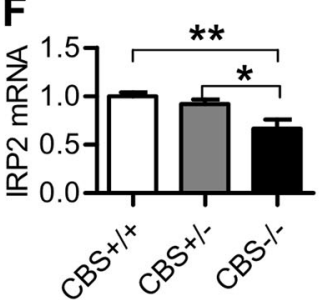

Fig. 5 CBS deficiency down-regulated expression of HIF-2a, EPO and IRPs in the liver. The expression of CBS (a), HIF-2a (b, d), EPO (c, d) proteins in the liver of $\mathrm{CBS}^{+/+}, \mathrm{CBS}^{+/-}$, and $\mathrm{CBS}^{-/-}$mice was examined by Western blot and immunofluorescence $(\times 400)$ analysis and IRP 1 (e) \& IRP2 (f) mRNAs by Real-time PCR analysis as described in the "Materials and Methods" section. Data are means \pm SEM $(n=6) .{ }^{*} p<0.05,{ }^{* *} p<0.01$, ${ }^{* * *} p<0.001$ vs. $\mathrm{CBS}^{+/-}$or $\mathrm{CBS}^{+/+}$mice

for hepcidin ${ }^{31}$. Hepcidin is a secreted peptide hormone encoded by the HAMP (hepcidin antimicrobial peptide) gene and produced predominantly by the liver ${ }^{32}$. After binding with hepcidin, Fpn1 is internalized and subsequently degraded ${ }^{31}$. The contents of hepcidin were found to be significantly increased in the blood of $\mathrm{CBS}^{-1-}$ mice when compared with $\mathrm{CBS}^{+/+}$or $\mathrm{CBS}^{+/-}$mice. The significant increase in hepcidin in $\mathrm{CBS}^{-1-}$ mice could lead to a significant reduction in Fpn1 and iron export from cells, which may also contribute to the increased iron content in the erythroblasts and bone marrow (Fig. 6). Also, TfR1 has been found to be directly inhibited by hepcidin in different types of cells ${ }^{33,34}$. Therefore, the increase in hepcidin is highly likely to be one of the causes of the reduced expression of TfR1 in the bone marrow (Fig. 6).

Increased expression of IL-6 will activate the JAK2/ STAT2 pathway, leading to an increase in the expression of hepcidin ${ }^{35}$. In addition, the increased expression of hepcidin may also be partly due to the reduced expression of ERFE in $\mathrm{CBS}^{-1-}$ mice. ERFE is a glycoprotein with 326amino-acid residues and has recently been identified as another regulator of iron homeostasis ${ }^{36}$. ERFE is produced by erythrocytic progenitors in response to EPO to increase iron absorption and mobilization of iron from stores by down-regulating hepcidin expression ${ }^{37}$. The reduced expression of EFRE in $\mathrm{CBS}^{-1-}$ mice would remove the inhibitory effects of EFRE on hepcidin expression, leading to an increase in the expression of hepcidin (Fig. 6).

In summary, our findings firstly, provide solid evidence to support the idea that CBS is essential for hemoglobin synthesis and erythropoiesis, and also demonstrate for the first time that CBS deficiency suppresses erythropoiesis, mainly by disrupting the expression of ALAS2 and FECH, two key enzymes involved in the heme biosynthetic 


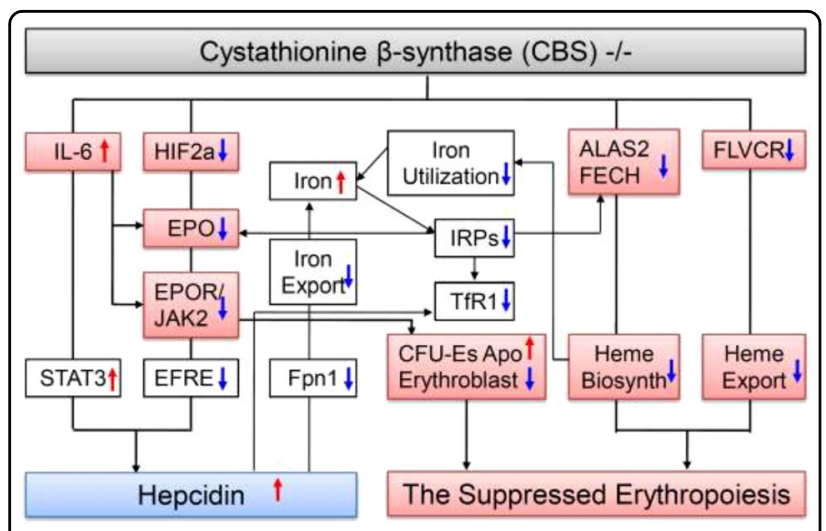

Fig. $6 \mathrm{~A}$ hypothetical scheme for the mechanisms involved in the suppressed erythropoiesis induced by CBS deficiency. CBS deficiency suppressed erythropoiesis mainly by disrupting expression of heme biosynthetic enzymes and transporter. CBS deficiency could induce a significant reduction in the expression of ALAS2 and FECH, two key enzymes involved in the heme biosynthetic pathway, and FLVCR, an exporter of heme from the cytosol and mitochondria, inhibiting heme biosynthesis and transport of heme from the cytosol and mitochondria in the bone marrow. Also, CBS deficiency could down-regulate HIF-2 $a$ and up-regulate IL-6 expression, and then inhibit EPO expression, and the reduced expression in EPO could increase apoptosis of CFU-Es and reduce the number of erythroblasts, which may also contribute to the suppressed erythropoiesis in CBS deficiency mice. In addition to the reduced iron utilization, the increased iron contents in the erythroblasts and bone marrow may be partly associated the reduced Fpn1 expression and then iron release from the bone marrow cells in $\mathrm{CBS}^{-1-}$ mice. The up-regulation of hepcidin in $\mathrm{CBS}^{-1-}$ mice may be due to the increased expression of IL-6 (IL-6/STAT3 pathway) and reduced contents of HIF2a/EFRE (HIF2a/ EPO/EPOR/EFRE pathway). (ALAS2 delta-aminolevulinate synthase, CBS Cystathionine $\beta$-synthase, EPO Erythropoietin, EPOR EPO receptor, FECH Ferrochelatase, FLVCR Feline leukemia virus subgroup $C$ cellular receptor, Fpn1 Ferroportin 1, HIF-2a Hypoxia inducible factor-2 subunit a, IL-6 Interleukin-6, IRPs Iron regulatory proteins, p-JAK2 Phospho-Janus Kinase 2, STAT3 Signal transducer and activator of transcription 3, TfR1 Transferrin receptor 1)

pathway, as well as FLVCR, an exporter of heme from the cytosol and mitochondria. Secondly, our data show that reduced EPO-induced increase in 'CFU-Es' apoptosis and the reduction in the number of erythroblasts may also be one of causes for the suppressed erythropoiesis in $\mathrm{CBS}^{-1-}$ mice. Thirdly, our results imply that, in addition to the reduced iron utilization, the increased iron content in erythroblasts and bone marrow may be partly associated with reduced Fpn1 expression and iron release from bone marrow cells in $\mathrm{CBS}^{-1-}$ mice. Finally, our study suggests that the up-regulation of hepcidin in $\mathrm{CBS}^{-1-}$ mice may be due to the increased expression of IL-6 and reduced HIF2a/EFRE contents (Fig. 6). These findings constitute important contributions to the knowledge of how our body can maintain iron homeostasis and may also be critical in elucidating the causes of patients with suppressed erythropoiesis.

\section{Materials and methods}

\section{Materials}

Unless otherwise stated, all chemicals were obtained from the Sigma Chemical Company, St. Louis, MO, USA. Mouse monoclonal anti-rat TfR1 was purchased from Invitrogen, Carlsbad, CA, USA; rabbit polyclonal antiFpn1 from Novus Biologicals, Littleton, CO, USA; mouse anti-p-JAK2 (phospho-Janus Kinase 2) from Cell Signaling Technology, Boston, USA; TRIzol reagent from Life Technologies, Carlsbad, CA, USA; and AevertAid First Strand cDNA Synthesis Kit from Thermo Scientific, Waltham, MA, USA. Rabbit polyclonal anti-Ft-Land antiDMT1 were purchased from Protein-tech, Chicago, IL, USA; rabbit polyclonal anti-CBS, HIF- $2 \alpha$ and EPO antibodies from Abcam, Inc., Cambridge, UK; rabbit polyclonal anti-Ft-H from Bioworld Technology Inc., Louis Park, MN, USA; goat anti-rabbit or anti-mouse IRDye $800 \mathrm{CW}$ secondary antibody from LI-COR Bio Sciences, Lincoln, NE, USA; FastStart Universal SYBR Green Master and LightCycler96 from Roche, Nutley, NJ, USA; and BCA protein Assay kit and protein RIPA lysis buffer from Beyotime Institute of Biotechnology, Haimen, JS, China.

\section{Animals}

The CBS heterozygous knockout C57BL/6 mice $\left(\mathrm{CBS}^{+/-}\right)$ were obtained from Professor Rui Wang of Lakehead University (Thunder Bay, ON, Canada). The $\mathrm{CBS}^{-1-}$, $\mathrm{CBS}^{+/-}$, and $\mathrm{CBS}^{+/+}$(wild-type) mice were verified by RT-PCR ${ }^{6}$. The mice were housed under specific pathogen-free conditions at $22 \pm 2{ }^{\circ} \mathrm{C}$ with a relative humidity of $60-65 \%$ and maintained under a 12-h light/ 12-h dark cycle with ad libitum access to food and water as previously described ${ }^{38}$. All animal care and experimental protocols were performed according to the Animal Management Rules of the Ministry of Health of China, and approved by the Animal Ethics Committees of Fudan University (NDFC31271132) and The Chinese University of Hong Kong (GRF14111815).

\section{Sampling of blood and tissues}

Animals were anesthetized with $1 \%$ pentobarbital sodium ( $40 \mathrm{mg} / \mathrm{kg}$ body weight, intraperitoneally) and decapitated. Blood samples were collected into syringes containing EDTA-2K for the determination of $\mathrm{RBC}, \mathrm{Hb}$, $\mathrm{HCT}, \mathrm{MCH}, \mathrm{MCV}$ as described previously ${ }^{39}$, dropped on the slide, and fixed with methanol and stained using Wright-Giemsa Stain after being pushed forward to the other end of the slide. Mice were perfused with phosphate-buffered saline (PBS), and the liver, spleen and kidney were removed, rinsed in PBS, dried, weighed, and stored in a freezing chamber at $-70{ }^{\circ} \mathrm{C}$ for subsequent measurements ${ }^{40,41}$. The femur was harvested, cut on one side, and bone marrow was pressed on the side of the slide 
and mixed with a small amount of fetal bovine serum according to Lozier andCalvo ${ }^{42}$.

\section{Bone marrow-derived macrophages}

BMDMs were prepared as described ${ }^{43}$ with some modifications. In brief, the bone was cut from the knee joint. Roswell Park Memorial Institute medium from a 10$\mathrm{mL}$ syringe with a 26-G needle was flushed into the joint and then collected. The entire medium was incubated with $\mathrm{NH} 4 \mathrm{Cl}$ solution on ice for $10 \mathrm{~min}$ to remove red blood cells, which were then collected for centrifuging at $1000 \mathrm{rmp}$ at $4{ }^{\circ} \mathrm{C}$ for $5 \mathrm{~min}$. Then, BMDMs were washed, cultured in differentiation medium (Roswell Park Memorial Institute medium with $40 \mathrm{ng} / \mathrm{mL}$ macrophage colony-stimulating factor), and incubated in fresh medium every $2-3$ days.

\section{Western blot analysis}

The tissues were washed and homogenized by protein RIPA lysis buffer as described previously ${ }^{44,45}$. Soluble proteins were collected after centrifugation at $13200 \mathrm{rpm}$ for $15 \mathrm{~min}$ at $4{ }^{\circ} \mathrm{C}$ and protein content was determined using the BCA protein assay reagent kit. Aliquots of the extract containing $40 \mu \mathrm{g}$ of protein were loaded and run on a single track of $10 \%$ SDS-PAGE and then transferred onto a pure nitrocellulose membrane (Bio-Rad). The blots were blocked with 5\% non-fat milk and then incubated overnight at $4^{\circ} \mathrm{C}$ with primary antibodies: rabbit anti-CBS (1:1,000), mouse anti-TfR1 (1:500), rabbit anti-DMT1 $(1: 1,000)$, rabbit anti-Fpn1 (1:1,000), rabbit anti-Ft-L $(1: 1,000)$, rabbit anti-Ft-H $(1: 1,000)$, rabbit anti-EPO (1:100), and rabbit anti-HIF-2 $\alpha$ (1:500). After being washed three times, the blots were incubated with goat anti-rabbit (1:1000) or anti-mouse IRDye800 CW secondary antibody (1:5000) for $2 \mathrm{~h}$ at room temperature. The intensities of the specific bands were detected and analyzed by the Odyssey infrared imaging system (Li-Cor, Lincoln, NE, USA). Anti- $\beta$-actin $(1: 2,000)$ was used as internal protein controls.

\section{Isolation of total RNA and quantitative real-time PCR}

The extraction of total RNA and preparation of cDNA were performed using TRIzol reagent and the AevertAid FirstStrand cDNA Synthesis Kit respectively, in accordance with the instructions of the manufacturers. Realtime PCR was carried out by RT-PCR instrument (LC96, Roche, Switzerland) using Fast Start Universal SYBR Green Master and the Light Cycler96. The specific pairs of primers of mouse TfR1, DMT1, Fpn1, Ft-L, IRP1 (iron regulatory proteins 1 ), IRP2 (iron regulatory proteins 2 ), EPO, EPOR, ALAS2, FECH, FLVCR, ERFE, and $\beta$-actin, are listed in Supplementary Table 1. The cycle threshold value of each target gene was normalized to that of the $\beta$-actin $\mathrm{mRNA}^{46,47}$. Relative gene expression was calculated by the $2-\Delta \Delta \mathrm{Ct}$ method.

\section{Immunofluorescence staining}

Immunofluorescence staining was carried out as described $^{48,49}$. In brief, femurs decalcified in 10\% EDTA and all tissues were immersed in cryo-embedding medium and then sectioned into 10 - $\mathrm{mm}$ thick slices using a cryotome (Leica Microsystems, Wetzlar, Germany). Slices were blocked with $3 \%$ bovine serum albumin for $2 \mathrm{~h}$ and incubated overnight at $4{ }^{\circ} \mathrm{C}$ with primary antibodies: rabbit anti-HIF-2 $\alpha$ (1:50) and rabbit anti-EPO (1:10). After being washed with $0.01 \mathrm{M}$ PBS three times, the slides were incubated with Alex Fluor 488 or 594conjugated secondary antibodies for $1 \mathrm{~h}$ at room temperature and then with Hoechest $33342(5 \mathrm{mg} / \mathrm{ml})$ for $15 \mathrm{~min}$ at $37^{\circ} \mathrm{C}$. Negative controls received an identical treatment except for the primary antibody and showed no positive signal.

\section{Immunohistochemistry staining}

Hydrogen peroxide was used to quench endogenous peroxidase activity $^{50}$. Sections were immunohistochemically stained with mouse anti-TfR1 (1:50), rabbit anti-DMT1 (1:50), rabbit anti-Fpn1 (1:50) and rabbit anti-Ft-L (1:100), rabbit anti-Ft-H (1:100), anti-p-JAK2 (1:100), and counterstained with hematoxylin. Signal was detected using a DAB substrate following the manufacturer's recommendations. The immunostaining images were scanned randomly under a confocal fluorescence microscope and light microscope (Olympus, Tokyo, Japan) by a single investigator who was blind to sample identity.

\section{Prussian blue staining}

Prussian blue staining was performed using freshly prepared 5\% potassium hexacya-noferrate and $5 \%$ hydrochloric acid ${ }^{51}$. Sections were rinsed in water and counterstained with nuclear fast red, dehydrated, and covered. The images were observed under a light microscope (Olympus, Tokyo, Japan).

\section{Statistical analysis}

Statistical analysis was performed using one-way analysis of variance and Tukey's method was used for multiple pair-wise comparisons. All data are expressed as the mean \pm SEM. A probability value of $P<0.05$ was taken to be statistically significant.

\section{Acknowledgements}

The studies in our laboratories were supported by the National Natural Science Foundation of China (NSFC31571195), the Hong Kong Research Grants Council (HKRGC) - General Research Fund (14110418, 14167817, 14107616). 


\section{Author contributions}

Y.K. and Z.M.Q. conceived, organized and supervised the study; P.Z., Y.J.C. and Y.S. performed the experiments; P.Z. and C.Q. contributed to the analysis of data. Y.K., C.Q. and Z.M.Q. prepared and wrote the manuscript.

\section{Author details}

${ }^{1}$ Laboratory of Neuropharmacology, Fudan University School of Pharmacy, Shanghai 201203, China. ${ }^{2}$ National Clinical Research Center for Aging and Medicine, Huashan Hospital, FudanUniversity, Shanghai 200040, China. ${ }^{3}$ School of Biomedical Sciences and Gerald Choa Neuroscience Centre, Faculty of Medicine, The Chinese University of Hong Kong, Shatin, NT, Hong Kong. ${ }^{4}$ Institute of Translational \& Precision Medicine, Nantong University, Nantong, JS 226019, China

\section{Conflict of interest}

The authors declare that they have no conflict of interest.

\section{Publisher's note}

Springer Nature remains neutral with regard to jurisdictional claims in published maps and institutional affiliations.

Supplementary Information accompanies this paper at (https://doi.org/ 10.1038/s41419-019-1951-0).

Received: 28 May 2019 Revised: 8 August 2019 Accepted: 26 August 2019 Published online: 24 September 2019

\section{References}

1. Yao, K. Effects of several unusual sulfur-containing amino acids on rat liver cystathionine-gamma-lyase. Physiol. Chem. Phys. 7, 401-408 (1975).

2. Fiedler, H. \& Wood, J. L. Specificity studies on the beta-mercaptopyruvatecyanide transsulfuration system. J. Biol. Chem. 222, 387-397 (1956).

3. Shen, $X$. et al. Measurement of plasma hydrogen sulfide in vivo and in vitro. Free Radic. Biol. Med. 50, 1021-1031 (2011).

4. Wang, R. Physiological implications of hydrogen sulfide: a whiff exploration that blossomed. Physiol. Rev. 92, 791-896 (2012)

5. Kimura, H. Signaling molecules: hydrogen sulfide and polysulfide. Antioxid. Redox Signal. 22, 362-376 (2015).

6. Zhou, Y. F. et al. Cysthionine $\beta$-synthase is required for body iron homeostasis. Hepatology 67, 21-35 (2018).

7. Isidori, A. et al. Iron toxicity -its effect on the bone marrow. Blood Rev. 32, 473-479 (2018).

8. Bessis, M. C. \& Breton-gorius, J. Iron metabolism in the bone marrow as seen by electron microscopy: a critical review. Blood 19, 635-663 (1962).

9. Leimberg, M. J., Prus, E., Konijn, A. M. \& Fibach, E. Macrophages function as a ferritin iron source for cultured human erythroid precursors. J. Cell. Biochem. 103, 1211-1218 (2008).

10. Orkin, S. H. \& Zon, L. I. Hematopoiesis: an evolving paradigm for stem cell biology. Cell 132, 631-644 (2008).

11. Nandakumar, S. K., Ulirsch, J. C. \& Sankaran, V. G. Advances in understanding erythropoiesis: evolving perspectives. Br. J. Haematol. 173, 206-218 (2016).

12. Zivot, A., Lipton, J. M., Narla, A. \& Blanc, L. Erythropoiesis: insights into pathophysiology and treatments in 2017. Mol. Med. 24, 11 (2018).

13. Kafina, M. D. \& Paw, B. H. Intracellular iron and heme trafficking and metabolism in developing erythroblasts. Metallomics 9, 1193-1203 (2017).

14. Iolascon, A., De Falco, L. \& Beaumont, C. Molecular basis of inherited microcytic anemia due to defects in iron acquisition or heme synthesis. Haematologica 94, 395-408 (2009).

15. Quigley, J. G. et al. Identification of a human heme exporter that is essential for erythropoiesis. Cell 118, 757-766 (2004).

16. Keel, S. B. et al. A heme export protein is required for red blood cell differentiation and iron homeostasis. Science 319, 825-828 (2008).

17. Lee, S. H. et al. Isolation and immunocytochemical characterization of human bone marrow stromal macrophages in hemopoietic clusters. J. Exp. Med. 168, 1193-1198 (1988).
18. Brasse-Lagnel, $C$. et al. Intestinal DMT1 cotransporter is down-regulated by hepcidin via proteasome internalization and degradation. Gastroenterology 140, 1261-1271.e1 (2011).

19. Singbrant, S. et al. Erythropoietin couples erythropoiesis, B-lymphopoiesis, and bone homeostasis within the bone marrow microenvironment. Blood 117, 5631-5642 (2011)

20. Jelkmann, W. Erythropoietin. Front. Horm. Res. 47, 115-127 (2016).

21. Rankin, E. B. et al. Hypoxia-inducible factor-2 (HIF-2) regulates hepatic erythropoietin in vivo. J. Clin. Invest. 117, 1068-1077 (2007).

22. Mastrogiannaki, M. et al. HIF-2alpha, but not HIF-1alpha, promotes iron absorption in mice. J. Clin. Invest. 119, 1159-1166 (2009).

23. Kapitsinou, P. P. et al. Hepatic HIF-2 regulates erythropoietic responses to hypoxia in renal anemia. Blood 116, 3039-3048 (2010).

24. Wiesener, M. S. et al. Widespread hypoxia-inducible expression of HIF-2alpha in distinct cell populations of different organs. FASEB J. 17, 271-273 (2003).

25. Haase, V. H. Regulation of enythropoiesis by hypoxia-inducible factors. Blood Rev. 27, 41-53 (2013).

26. Paliege, A. et al. Hypoxia-inducible factor-2alpha-expressing interstitial fibroblasts are the only renal cells that express erythropoietin under hypoxiainducible factor stabilization. Kidney Int. 77, 312-318 (2010).

27. Pierce, C. N. \& Larson, D. F. Inflammatory cytokine inhibition of erythropoiesis in patients implanted with a mechanical circulatory assist device. Perfusion $\mathbf{2 0}$ 83-90 (2005)

28. Leng, H. M., Kidson, S. H., Keraan, M. M., Randall, G. W. \& Folb, P. I. Cytokinemediated inhibition of erythropoietin synthesis by dexamethasone. J. Pharm. Pharmacol. 48, 971-974 (1996).

29. Wang, C. Q., Udupa, K. B. \& Lipschitz, D. A. Interferon-gamma exerts its negative regulatory effect primarily on the earliest stages of murine erythroid progenitor cell development. J. Cell. Physiol. 162, 134-138 (1995).

30. Taniguchi, S., Dai, C. H., Price, J. O. \& Krantz, S. B. Interferon gamma downregulates stem cell factor and erythropoietin receptors but not insulin-like growth factor-I receptors in human erythroid colony-forming cells. Blood 90, 2244-2252 (1997)

31. Nemeth, E. et al. Hepcidin regulates cellular iron efflux by binding to ferroportin and inducing its internalization. Science 306, 2090-2093 (2004).

32. Hentze, M. W., Muckenthaler, M. U., Galy, B. \& Camaschella, C. Two to tango: regulation of Mammalian iron metabolism. Cell 142, 24-38 (2010).

33. Du, F. et al. Hepcidin directly inhibits transferrin receptor 1 expression in astrocytes via a cyclic AMP-protein kinase A pathway. Glia 59, 936-945 (2011).

34. Du, F., Qian, Z. M., Luo, Q., Yung, W. H. \& Ke, Y. Hepcidin suppresses brain iron accumulation by downregulating iron transport proteins in iron-overloaded rats. Mol. Neurobiol. 52, 101-114 (2015).

35. Qian, Z. M. et al. Lipopolysaccharides upregulate hepcidin in neuron via microglia and the IL-6/STAT3 signaling pathway. Mol. Neurobiol. 50, 811-820 (2014).

36. Kautz, L., Jung, G., Nemeth, E. \& Ganz, T. Erythroferrone contributes to recovery from anemia of inflammation. Blood 124, 2569-2574 (2014).

37. Robach, P. et al. Serum hepcidin levels and muscle iron proteins in humans injected with low- or high-dose erythropoietin. Eur. J. Haematol. 91, 74-84 (2013).

38. Qian, Z. M., Xiao, D. S., Ke, Y. \& Liao, Q. K. Increased nitric oxide is one of the causes of changes of iron metabolism in strenuously exercised rats. Am. J. Physiol. 280, R739-R743 (2001).

39. Qian, Z. M., Xiao, D. S., Tang, P. L., Yao, F. Y. \& Liao, Q. K. Increased expression of transferrin receptor on membrane of erythroblasts in strenuously exercised rats. J. Appl. Physiol. 87, 523-529 (1999).

40. Xiao, D. S., Ho, K. P. \& Qian, Z. M. Nitric oxide inhibition decreases bleomycindetectable iron in spleen, bone marrow cells and heart but not in liver in exercise rats. Mol. Cell. Biochem. 260, 31-37 (2004).

41. Ke, Y. et al. Post-transcriptional expression of DMT1 in the heart of rat. J. Cell. Physiol. 196, 124-130 (2003).

42. Lozier, J. N. \& Calvo, K. R. Effect of viral decontamination measures on Wrightstained blood smears. Blood 125, 1350-1351 (2015).

43. Meynard, D. et al. Lack of the bone morphogenetic protein BMP6 induces massive iron overload. Nat. Genet. 41, 478-481 (2009).

44. $\mathrm{Yu}, \mathrm{S}$. et al. La3+-promoted proliferation is interconnected with apoptosis in $\mathrm{NIH} 3 \mathrm{T3}$ cells. J. Cell. Biochem. 94, 508-519 (2005).

45. Chang, Y. Z. et al. Increased divalent metal transporter 1 expression might be associated with the neurotoxicity of L-DOPA. Mol. Pharmacol. 69, 968-974 (2006). 
46. Chen, $Y$. et al. Iron loading inhibits ferroportin1 expression in PC12 cells. Neurochem. Int. 47, 507-513 (2005).

47. Huang, X. T. et al. Reducing iron in the brain: a novel pharmacologic mechanism of huperzine A in the treatment of Alzheimer's disease. Neurobiol. Aging 35, 1045-1054 (2014).

48. Du, F. et al. Purity, cell viability, expression of GFAP and bystin in astrocytes cultured by different procedures. J. Cell. Biochem. 109, 30-37 (2010).

49. Du, F. et al. Hyperthermic preconditioning protects astrocytes from ischemia/reperfusion injury by up-regulation of HIF-1 alpha expression and binding activity. Biochim. Biophys. Acta 1802 1048-1053 (2010).

50. Li, L. et al. Complement C $5 \mathrm{a}$ is detrimental to histological and functional locomotor recovery after spinal cord injury in mice. Neurobiol. Dis. 66, 74-82 (2014).

51. Millward, J. M. et al. Iron oxide magnetic nanoparticles highlight early involvement of the choroid plexus in central nervous system inflammation. ASN Neuro. 5, e00110 (2013). 